\title{
Collaborative Care Drives Triple Aim Success in Patients With Uncontrolled Hypertension
}

Melanie McMinn, MD; Greg Weidner, MD, FACP; Angie Stevens, RN, CDE; Monique Lucas, RN, BSN, CCM, CCTM

Carolinas Healthcare System, Proactive Health, Charlotte, NC, United States

Corresponding Author:

Melanie McMinn, MD

Carolinas Healthcare System

Proactive Health

15110 John J Delaney Dr

Ste 200

Charlotte, NC, 28277

United States

Phone: 17043028100

Fax: 17043028101

Email: melanie.mcminn@carolinashealthcare.org

\section{Abstract}

Background: We are an outpatient internal medicine practice that has developed a team-based technology-enabled approach to managing chronic medical conditions.

Objective: To develop a collaborative approach to managing chronic conditions that is patient-centric, while achieving better outcomes at a lower cost.

Methods: We chose to focus on patients with hypertension. Target patients were identified by partner referrals and by our own data registry reports of patients with blood pressure >140/90. Individuals were then invited to participate in a 90-day program with the focused intent of actively engaging the patients in managing their blood pressure. The invitations were either conducted in person, sent via a patient portal, or sent in a mailed letter. Each interested patient was assigned a health advocate and asked to record their blood pressure in an online application daily. There was no charge to the patient. Individual goals were determined by the patient, with the guidance of the physician and health advocate using a shared decision-making tool, and strategy focused on a habit-based behavior change model. Patients either purchased a Bluetooth-enabled digital blood pressure cuff from our office or were permitted to use their own blood pressure cuff, which was calibrated by our nursing staff. Patients communicated with their health care team via an online shared message board as well as through a patient portal. Virtual visits were conducted as needed throughout the 90-day program with either the physician, health advocate, or dietician. Both curated and created educational materials were shared with the patient. Health coaching was ongoing, and communication frequency and modality preference was collaboratively determined by the patient and care team.

Results: Overall, we invited 920 patients to participate in our program. Of these, 224 patients enrolled and completed the 90 -day program. We tracked metrics including blood pressure values, frequency of in-office and virtual visits, time spent by all team members engaged in the online platform, and money saved by averting office visits. Our hypertension patients visited the primary care office half as often as traditionally managed hypertension patients and achieved their target blood pressure faster. In standard hypertension care, research would tell us that $30 \%$ of patients will have a normal blood pressure in 1 year. In this study, $80 \%$ of our patients had a controlled blood pressure in 3 months. In the few cases where blood pressure control could not be achieved with the above-mentioned methods, a search for secondary causes of hypertension was initiated by the primary care physician. We also demonstrated US $\$ 350$ in primary care direct cost savings annually by avoiding an average of 2 office visits over these 90 days. The successes, however, are not only improvement in cost and blood pressure values; $93 \%$ of patients in this program report a "very high quality" or "high quality" overall patient experience.

Conclusions: Our program achieves higher quality care at a lower cost with higher patient satisfaction than traditional care. We are confident that this approach to hypertension can be applied to other chronic disease states with equally as impressive results. 
(iproc 2016;2(1):e16) doi: 10.2196/iproc.6084

\section{KEYWORDS}

collaborative care; triple aim; hypertension; connected health; patient engagement

This poster was presented at the Connected Health Symposium is displayed as an image in Figure 1 and as a PDF in Multimedia 2016, October 20-21, Boston, MA, United States. The poster Appendix 1.

Figure 1. Poster.



\section{Multimedia Appendix 1}

Poster.

[PDF File (Adobe PDF File), 2MB-Multimedia Appendix 1]

Edited by T Hale; submitted 02.06.16; peer-reviewed by CHS Scientific Program Committee; accepted 08.08.16; published 22.12.16
Please cite as:
McMinn M, Weidner G, Stevens A, Lucas $M$
Collaborative Care Drives Triple Aim Success in Patients With Uncontrolled Hypertension
iproc 2016;2(1):e16
URL: $\underline{\text { http://www.iproc.org/2016/1/e16/ }}$
doi: $\underline{10.2196 / \text { iproc.6084 }}$
PMID:

CMelanie McMinn, Greg Weidner, Angie Stevens, Monique Lucas. Originally published in Iproceedings (http://www.iproc.org), 22.12.2016. This is an open-access article distributed under the terms of the Creative Commons Attribution License (http://creativecommons.org/licenses/by/2.0/), which permits unrestricted use, distribution, and reproduction in any medium, 
provided the original work, first published in Iproceedings, is properly cited. The complete bibliographic information, a link to the original publication on http://www.iproc.org/, as well as this copyright and license information must be included. 